\section{Continuity Laws for Oriented Polymers}

\section{Theo Odijk}

Department of Polymer Technology, Faculty of Chemical Engineering and Materials Science, Delft University of Technology, P.O. Box 5045, 2600 GA Delft, The Netherlands

Received April 10, 1992

Revised Manuscript Received June 22, 1992

Macromolecular chains are oriented in a wide range of materials. Important examples include polymer liquid crystals, grafted chains, ordered copolymers, polymer crystals, and fibers. Orientational order may occur on more than one level. For instance, in micellar systems the alkyl chains are oriented because they are densely packed, yet on a mesoscopic level semiflexible micelles themselves may align into nematic or hexagonal phases. Anisometric bundles of ordered chains are often formed when polarizable colloidal particles aggregate in strong external fields. Apparently, Flory was the first to recognize the way density couples to the degree of orientational order in a polymeric crystal. ${ }^{1}$ de Gennes expressed this as a differential conservation law in a discussion on lipid organization. ${ }^{2}$ In these problems the director has a uniform orientation in space. Later, de Gennes introduced a similar though not identical expression relating the segment density with the fluctuation in the director in a polymer nematic. ${ }^{3}$ Implicit in this analysis is the spatial invariance of the degree of orientational order. In general, however, an oriented polymer is described by three experimentally accessible fields depending on position $\vec{r}$ : the director $\dot{n}(\vec{r})$, the segment density $\rho(\vec{r})$, and the orientational order $S_{1}(\vec{r})$. The object of this paper is to establish their connection by a straightforward generalization of refs 2 and 3 .

For definiteness, we let the polymer sample consist of monodisperse chains which adopt wormlike configurations (a particular chain $\mathrm{i}$ is described by the radius vector $\vec{R}_{\mathrm{i}}\left(s_{\mathrm{i}}\right)$ with $s_{\mathrm{i}}$ the contour distance from one end; the contour length is $L$ ). They are fairly highly oriented so for the moment we assume they are directed; i.e., the sample is free of hairpins. Furthermore, the chains are so long that our sample contains no free ends, at least in the bulk. This idealized situation is depicted in Figure 1. This may be one particular realization in an ensemble (for instance, if the sample is in (local) thermal equilibrium as in a liquid crystal) or a quenched configuration (for instance, if our material is a fiber).

Next, we argue that a hydrodynamic description ${ }^{4}$ is possible. We spatially average over the microscopic variables in Figure 1 and retain appropriate macroscopic fields $\left(\vec{n}(\vec{r}), \rho(\vec{r}), S_{1}(\vec{r})\right)$ which vary slowly in comparison with the original variables. In order to render the hydrodynamic analogy exact, $N$ pointlike particles are allowed to flow from left to right along each curve in Figure 1. The particles are spaced equidistantly; ultimately, we wish to take the continuum limit with $N$ increasing without bound. For convenience we set the mass of each particle equal to unity. Finally, the particles are given a constant speed $w$. Accordingly, on the left, particles are created at a constant rate $w N L^{-1}$ per chain; on the right, they are annihilated at the same absolute rate.

We now introduce a macroscopic velocity field depending on time $t$ but which is stationary in the continuum limit.

$$
\vec{V}(\vec{r}, t) \equiv\left\langle\vec{v}_{\mathbf{k}}\right\rangle_{;}
$$

The volume average of the particle velocity $\vec{v}_{\mathrm{k}}$ is effected

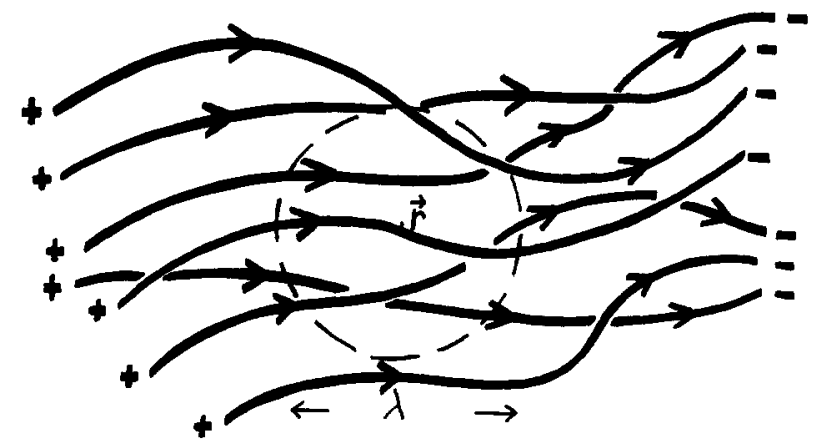

Figure 1. Oriented polymer consisting of wormlike chains without hairpins. Particles are added on the left $(+)$, flow in the direction of the arrows, and are removed on the right $(-)$.

over all $M_{\lambda}$ particles $\mathbf{k}$ enclosed within a volume $\Omega_{\lambda}$ of order $\lambda^{3}$ centered around point $\vec{r}$ (Figure 1 ). Such a coarse graining makes sense only if $\lambda$ greatly exceeds the typically rapid microvariation of the chains yet is much smaller than the scale of variation of the hydrodynamic field $\vec{V}(\vec{r}, t)$. In addition, we have $\vec{v}_{\mathrm{k}}=\partial \vec{R}_{\mathrm{i}}\left(s_{\mathrm{k}}(t)\right) / \partial t=w \partial \vec{R}_{\mathrm{i}}\left(s_{\mathrm{k}}\right) / \partial s_{\mathrm{k}}$ 日 $w \vec{u}_{\mathrm{i}}\left(s_{\mathrm{k}}\right)$ for particle $\mathrm{k}$ situated at contour distance $s_{\mathrm{k}}$ on chain $i$. Hence, we have in the continuum limit

$$
V(\vec{r}, t)=V(\vec{r})=w\langle\vec{u}\rangle_{\vec{r}} \equiv w \vec{U}(\vec{r})
$$

where the volume average is now over all unit vectors $\vec{u}$ within $\Omega_{\lambda}$.

In a similar fashion we define a macroscopic number density of particles

$$
d(\vec{r}, t)=\frac{M_{\lambda}(\vec{r})}{\Omega_{\lambda}(\vec{r})}
$$

provided $\lambda$ is again an appropriate mesoscopic scale. The density $d(\vec{r}, t)$ becomes essentially a stationary quantity $d(\vec{r})$ as $N$ becomes larger and larger. In the continuum limit we have for the segment density $\rho(\vec{r}) \equiv L d(\vec{r}) / N A$ with $A$ the segment length.

We may now write an equation of continuity for our fluid of particles. ${ }^{4}$

$$
\partial d / \partial t+\vec{\nabla} \cdot(d \vec{V})=0
$$

In the continuum limit $(N \rightarrow \infty)$, we have $\partial d / \partial t=0$, so eq 4 implies

$$
\vec{\nabla} \cdot(\rho \vec{U})=0
$$

for the macroscopic fields $\rho(\vec{r})$ and $\vec{U}(\vec{r})$ of the oriented polymer.

The field $\vec{U}(\vec{r})$ by itself is an elusive quantity. Hence, it is expedient to introduce the director $\vec{n}(\vec{r})$ and the variable $S_{1}(\vec{r})$ defining the degree of segment order with respect to the director. Both $\vec{n}$ and $S_{1}$ are independently measurable fields.

$$
\langle\vec{u}\rangle_{\vec{r}}=\vec{U}(\vec{r}) \equiv S_{1}(\vec{r}) \vec{n}(\vec{r})
$$

In view of the fact that $\tilde{n}^{2}=1$, we have

$$
S_{1}(\vec{r}) \equiv\langle\vec{n}(\vec{r}) \cdot \vec{u}\rangle_{\dot{r}}
$$

Equation 5 becomes

$$
\vec{\nabla} \cdot\left(\rho S_{1} \vec{n}\right)=0
$$

which expresses a connection between three experimentally attainable fields and generalizes refs 2 and 3 . It is important to stress that $\vec{n}(\vec{r})$ is here a polar vector field, by definition. Thus, $S_{1}(\vec{r})$ is not to be confused with the usual order parameter. 

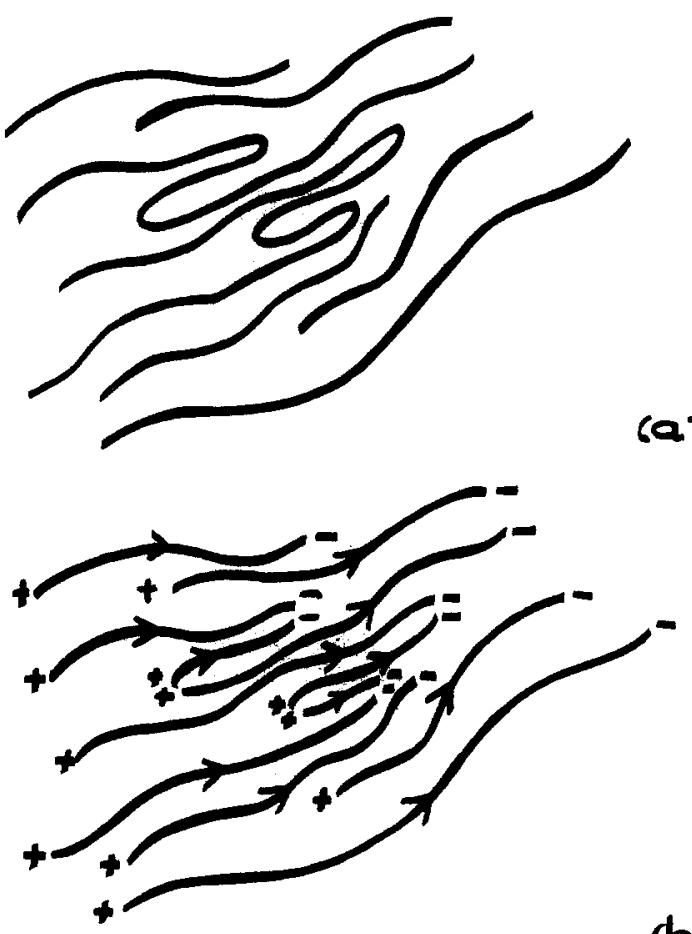

b)

Figure 2. (a) Oriented polymer containing hairpins and chain ends in the bulk. (b) Equivalent sample of directed chains; particles flow as in Figure 1.

For a highly oriented polymer it is possible to simplify eq 8 further. First, in a large class of materials the polymer is uniaxially ordered so we may introduce an orientational distribution function $f(\vec{r}, \vec{u} \cdot \vec{n}(\vec{r}))$. Next the distribution is sharply peaked and may be approximated by a Gaussian

$$
\begin{gathered}
f(\theta) \sim \frac{\alpha(\vec{r})}{2 \pi} \exp \left[-(1 / 2) \alpha(\vec{r}) \theta^{2}\right] \quad(0 \leq \theta \leq(1 / 2) \pi) \\
S_{1}(\vec{r}) \sim 1-\alpha^{-1}(\vec{r})
\end{gathered}
$$

where $\vec{u} \cdot \vec{n}(\vec{r}) \equiv \cos \theta \simeq 1-(1 / 2) \theta^{2}$ with $\theta$ the angle between an infinitesimal segment and the director. The parameter $\alpha(\vec{r})$ is much larger than unity and is postulated to vary slowly on the scale of $\lambda$. Equation 9 may be interpreted in two ways: (1) the vector $(\vec{u}-\vec{n})$ is a superposition of independent random vectors so that the central limit theorem holds; (2) eq 9 is a Boltzmann distribution of a chain segment in a harmonic potential which itself is a second-order Taylor expansion of some mean potential. Hence, eq 8 reduces to the expression

$$
\vec{\nabla} \cdot\left[\rho\left(1-\alpha^{-1}\right) \vec{n}\right]=0
$$

A readily accessible quantity is the birefringence, which in this case is proportional to the usual order parameter $S_{2}(\vec{r})=\left\langle P_{2}(\cos \theta)\right\rangle_{r}$. Equation 11 is conveniently rewritten in terms of $S_{2}$

$$
\vec{\nabla} \cdot\left[\rho\left(S_{2}+2\right) \dot{n}\right]=0
$$

It is illuminating to analyze the fluid of particles in the context of kinetic theory. We introduce a distribution function ${ }^{5} f(\vec{r}, \vec{v}, t)$ in terms of the generalized coordinates $\vec{r}$ of a particle and its generalized momentum $\vec{v}$. The transport equation is ${ }^{5}$

$$
\frac{\mathrm{d} f}{\mathrm{~d} t}=\frac{\partial f}{\partial t}+\vec{v} \cdot \vec{\nabla} f+A(\vec{r}) \vec{v} x \vec{n}(\vec{r}) \frac{\partial f}{\partial \vec{v}}=C(f)
$$

where the total time derivative is defined along the phase path. Here, $C(f)$ represents a collision integral. If $C$ were zero, the gas would be collisionless and Liouville's theorem ${ }^{5}$ would hold $(\mathrm{d} f / \mathrm{d} t=0)$. In our case, there is an external force $A(\vec{r}) \vec{u} \times \vec{n}(\vec{r})$ which must be perpendicular to $\vec{n}$ because it represents the way particles accommodate to the changing director but also perpendicular to $\dot{v}$ since the particles flow along wormlike curves at constant speed so that the magnitude of their momentum is conserved. If there were no collisions, our fluid would behave almost like a collisionless gas of electrons in a spatially varying magnetic field. ${ }^{10}$ Note that the term involving the external force vanishes for a uniaxial distribution $f(\vec{r}, \vec{v} \cdot \vec{n}(\vec{r}), t)$. In general, $C(f)$ is exceedingly complicated. If the chains were ideal, the particles would still follow wormlike paths. In the annealed case $C(f)$ would reduce to a term proportional to $\partial^{2} f / \partial \vec{v}^{2}$ (see ref 5). In the quenched case we might introduce a second gas of hypothetical particles which collide with the original particles so that they do follow the prescribed paths at constant speed. A systematic theory of colliding chains or paths is difficult to set up.

Whatever the form of $C(f)$, particles are not lost by collisions, although they may change the direction of their momentum. An integration of eq 13 over all $\bar{v}$ then yields

$$
\int \mathrm{d} \vec{v} \vec{v} \cdot \vec{\nabla} f=0
$$

It can be shown that the term involving the external force vanishes. Furthermore, in the continuum limit the distribution is stationary $(\partial f / \partial t=0)$. Defining the reduced distribution $d(\vec{r})$ and the hydrodynamic velocity field $\vec{V}(\vec{r})$

$$
\begin{aligned}
& d(\vec{r}) \equiv \int \mathrm{d} \vec{v} f(\vec{r}, \vec{v}) \\
& \vec{V} \equiv \frac{\int \mathrm{d} \vec{v} \vec{v} f(\vec{r}, \vec{v})}{\int \mathrm{d} \vec{v} f(\vec{r}, \vec{v})}
\end{aligned}
$$

we thus regain the stationary form of the continuity equation (4) from the transport equation (13).

Equations 8 and 12 are valid for a sample free of chain ends and hairpins. In the general case in Figure $2 a$ we can again set up a hydrodynamic theory for directed chains provided we break up the hairpins in the manner shown in Figure 2b. If particles are allowed to flow along the chains as before, we now have to create and annihilate them in the bulk as well. The total density of + ends where particles are formed is $\rho_{+}(\vec{r})$, which may differ from $\rho_{-}(\vec{r})$, the total density of - ends where particles are destroyed. The rates of creation and annihilation per end are identical. It is straightforward to show that the equation of continuity is modified to

$$
\vec{\nabla} \cdot\left(\rho S_{i} \vec{n}\right)=A^{-1}\left(\rho_{+}-\rho_{-}\right)
$$

This generalizes an expression first derived by Meyer ${ }^{6}$ for perfectly aligned rods in a splay field (i.e., with $S_{1} \equiv 1$ ). For related discussions, see refs 7-9. Equation 17 allows us to estimate when we may disregard chain defects.

Equations 8 and 12 should be useful in analyzing the degree of orientational order in a well-aligned polymer sample free from macroscopic defects. The director $\tilde{n}(\vec{r})$ will often be fixed by the confining geometry, and $\rho(\vec{r})$ is proportional to the absorption of suitable radiation. In particular, eq 12 would then yield an estimate of the spatial variation in the birefringence for a highly oriented polymer.

\section{References and Notes}

(1) Flory, P. J. J. Am. Chem. Soc. 1962, 84, 2857.

(2) de Gennes, P.-G. Phys. Lett. 1974, 47A, 123.

(3) de Gennes, P.-G. Mol. Cryst. Liq. Cryst. 1977, 34, 177. 
(4) Landau, L. D.; Lifshitz, E. M. Fluid Mechanics; Pergamon: London, 1959.

(5) Lifshitz, E. M.; Pitsevskii, L. P. Physical Kinetics; Pergamon: Oxford, 1981.

(6) Meyer, R. B.In Polymer Liquid Crystals; Ciferri, A., Krigbaum, W. R., Meyer, R. B., Eds.; Academic: New York, 1982.

(7) Vroege, G. J.; Odijk, T. Macromolecules 1988, 21, 2848.

(8) Le Doussal, P.; Nelson, D. R. Europhys. Lett. 1991, 15, 161.

(9) Selinger, J. V.; Bruinsma, R. Preprint.
(10) In a magnetoactive plasma, the electric field may be negligible so that a Lorentz force proportional to $\vec{v}_{0} X \vec{B}(\vec{r})$ is ererted on an electron moving with velocity $\vec{v}_{\mathrm{e}}$ in a slowly varying magnetic field $B(\vec{r})$. Furthermore, if the plosma is highly conducting, the magnetic lines of force are frozen in the fluid and $B(F)$ is aligned along the hydrodynamic velocity field $V_{\circ}(\vec{F})$ or vice versa (see ref 5). The analogy $\left(\vec{v} \leftrightarrow \dot{v}_{s} ; \vec{n}(\vec{F}) \leftrightarrow B(\vec{r}) ; \vec{V}(\vec{r}) \rightarrow\right.$ $\left.\vec{V}_{\theta}(\vec{r})\right)$ is not exact because, here, $\vec{v}$ has a constant magnitude $w$. 